\title{
Pathophysiologic and Prognostic Role of IL-10 as a Proinflammatory and Regulatory Cytokine in Dengue Fever
}

\author{
Saishruti $^{1}$, G. Sucila Thangam ${ }^{2} *$ and Revathy $^{1}$ \\ ${ }^{1}$ Department of Microbiology, Tirunelveli Medical College, Tirunelveli - 627011 , \\ Tamil Nadu, India \\ ${ }^{2}$ Department of Microbiology, Government Theni Medical College, Theni - 625512 , \\ Tamil Nadu, India \\ *Corresponding author
}

Keywords

Dengue shock syndrome, Dengue Hemorrhagic Fever, ELISA, Platelet count, IL-10

Article Info

Accepted:

12 December 2018 Available Online: 10 January 2019
A B S T R A C T

Dengue shock syndrome is a severe complication of Dengue Hemorrhagic Fever (DHF), characterized by a massive increase in vascular permeability. This prospective study was conducted to assess the pathophysiologic and prognostic role of proinflammatory and regulatory cytokines in the inpatient population tested positive for Dengue fever by using commercially prepared ELISA kits for IL-10. A total of 100 blood samples for this study were collected from pediatric patients and adult patients. The patients were tested for NS1 antigen of DENV and IgM antibodies against DENV. The levels of Positive cases were then selected for the study as per their platelet count. IL 10 was obtained using ELISA and statistically analyzed and results were interpreted. All the patients included in the study had a platelet count of less than 1,00,000/cu.mm. minimum count being 16000 and a maximum of 1,00,000 with a mean value of 63,000 . One third of the Dengue positive patients had very high levels of IL-10 level. Interleukin 10 values and clinical features were analyzed using Pearson's Chi Square Test and the Significance was calculated. There was a significant association $(\mathrm{p}=0.025)$ between the IL categorization and the clinical features i.e. patients with Severe Dengue or Dengue with danger signs had significantly higher number of IL-10 levels. Early diagnosis and interventional measures can play a vital role in Dengue prognosis and can also prevent the patient from landing into Dengue Hemorrhagic Fever or Dengue Shock Syndrome. The present study clearly indicates that IL10 is a highly sensitive marker of Severe Dengue and can be used as a screening tool in Secondary Dengue patients or those with warning signs.

\section{Introduction}

Dengue is the most rapidly spreading mosquito-borne viral disease in the world. During the last fifty years, incidence has risen to 30-fold with increasing geographic expansion to new countries and, in the present scenario, from urban to rural settings.

An estimated 50 million dengue infections occur every year and approximately 2.5 billion people are living in dengue endemic countries 
(Cecilia, D, 2014; Deen, et al., 2006) The 2002 World Health Assembly resolution 55.17 urged greater commitment to dengue by WHO and its Member States. Of particular significance is the 2005 World Health Assembly resolution 58.3 on the revision of the International Health Regulations (IHR), which includes dengue as an example of a disease that may cause a public health emergency of international concern with implications on health security due to disruption and rapid epidemic spread beyond national borders (WHO, 2009)

Some 1.8 billion (more than 70\%) of the population at risk for dengue worldwide live in member states of the WHO South-East Asia Region and Western Pacific Region, which bear nearly $75 \%$ of the current global disease burden due to Dengue. The Asia Pacific Dengue Strategic Plan for both regions was been prepared in consultation with member countries and development partners in response to the increasing threat from Dengue, which is spreading to new geographical areas and causing high mortality during the early phase of outbreaks. The strategic plan aims to aid countries to reverse the rising trend of dengue by enhancing their preparedness to detect, characterize and contain outbreaks rapidly and to stop the spread to new areas. Since 2000, epidemic Dengue has spread to new areas and has increased in the already affected areas of the region. In 2003, eight countries -- Bangladesh, India, Indonesia, Maldives, Myanmar, Sri Lanka, Thailand and Timor-Leste -- reported Dengue cases.

In India, changes in genotypes/lineages have been associated with increasing severity (WHO, 2009). Tirunelveli district (Tamilnadu) faced an epidemic in 2012 and 2013 at Kadayanallur and Ambasamudram blocks. Tirunelveli Medical College Hospital (TVMCH) is the only tertiary care centre in this district.
Dengue virus (DENV) is a positive-sense, single-stranded RNA virus in the family Flaviviridae that causes disease in human beings. DENV infection results in different clinical manifestations ranking from benign disease [Dengue fever (DF)] to severe disease [Dengue Hemorrhagic fever (DHF)] (Monath, 1994). In situations where the onset of hypovolaemic shock or dengue shock syndrome (DSS) persists, DHF can result in a life-threatening infection.

DHF is characterized by increased vascular permeability resulting in plasma leakage and coagulation derangements. DHF can be classified further into four degrees of severity, and degrees III and IV are considered to be DSS (WHO, 2009). Due to the lack of efficient biomarkers that define endothelial damage for determining the degree of severity, there is an urgent need to find relevant biological markers of disease, as well as to determine more coherent definitions of the degree of severity in patients (Natalia Houghton-Trivin et al., 2010).

\section{Case definition}

Severe Dengue - Dengue confirmed cases plus severe thrombocytopenia $(<50,000$ platelets/mm3) and/or hypotension (postural hypotension with decrease in systolic arterial pressure in $20 \mathrm{~mm} \mathrm{Hg}$ in supine position or systolic arterial pressure $<90 \mathrm{~mm} \mathrm{Hg}$ ) and/or plasma leakage (either haemoconcentration fluctuation of packed cell volume $\geq 20 \%$ during illness course and recovery or clinical signs of plasma leakage, such as pleural effusion) and/or severe haemorrhagic manifestations.

Mild dengue - Dengue confirmed cases in absence of severe thrombocytopenia, hypotension, plasma leakage signs or haemorrhagic manifestations. Viral and host factors can both contribute to disease severity. 
Epidemiologic data suggests that there is a greater risk of DHF/DSS during secondary infections, and immunopathologic mechanisms, such as immune-enhancement phenomenon, have been proposed to contribute to DHF risk. This contribution occurs when non neutralizing antibodies resulting from the primary infection favour dissemination of the second infecting Dengue virus, a phenomenon known as antibodydependent enhancement, and cross-reactive memory $\mathrm{T}$ cells from a primary infection recognize antigen from the secondary infection, resulting in increased $\mathrm{T}$ cell activation and cytokine production. Antibody enhancement, improper $\mathrm{T}$ cell and cytokine response and host genetic factors are amongst the postulated immunopathogenesis leading to severe dengue (Halstead, 2009; Martin, et al., 2011).

Cytokines are important immunomodulators and improper T-cell activation can lead to cytokine storms which are believed to result in endothelial permeability and leakage, a typical feature of Dengue disease progressing into a more severe stage. Many cytokines have been implied in causing severe Dengue manifestations among which IL-10 poses to be an important one. Increased levels of serum IL-10 may be a useful prognostic hallmark in DHF/DSS patients. IL-10 is a cytokine with pleiotropic effects in immunoregulation and inflammation. Higher levels of IL-10 are detected in DHF/DSS patients compared with DF patients, and this trend is observed for infants, children, and adults. Studies show increased levels of IL-10 from the onset of fever to defervescence, and viremia primarily occurs during fever in Dengue patients. The relationship between IL-10 and viral replication is therefore speculated. Targeting IL-10 regulation and signalling pharmacologically using neutralizing antibodies, antagonists, and inhibitors may represent a viable therapeutic strategy for preventing the progression of severe dengue diseases. It is believed that the critical point in Dengue physiopathology is the plasmatic leakage mediated by the host immunological response (Tsung et al., 2013).

\section{Materials and Methods}

This prospective study was conducted at Tirunelveli Medical College, Tamilnadu from March to June 2017 to assess the pathophysiologic and prognostic role of proinflammatory and regulatory cytokines in the inpatient population tested positive for Dengue fever by using commercially prepared ELISA kits for IL-10. The levels of IL 10 was obtained using ELISA and statistically analysed and results were interpreted.

Blood samples were obtained from those received at the central laboratory for Dengue serology. Children aged 4-12 years and adults 18-60 years with clinical signs suggestive of dengue admitted in Paediatric and Medicine wards were included in the study while patients with fever not admitted in the hospital or those with other known infectious illnesses (TB/ HIV/ HCV/ malaria/ Japanese encephalitis / Typhoid / Chikungunya) were excluded from the study. All the samples included were positive for Dengue $\operatorname{IgM}$ antibodies and NS1 protein; and with a platelet count of less than or equal to one lakh.

The blood samples were collected, transported, stored and processed using all safety precautions throughout the course of this study. The institutional ethical clearance was obtained for this study. NS1 ELISA was done using commercially available kits and Dengue IgM ELISA kits were obtained from NIV, Pune. The IL10 kits were those commercially obtained from Diaclone, France. The absorbance was read on a spectrophotometer using $450 \mathrm{~nm}$ as the primary wavelength and optimally $620 \mathrm{~nm}$ as the reference wave length. 


\section{Statistical analysis}

The demographic parameters, clinical features and platelet counts were correlated using ChiSquare test. Subsequently the cytokines and their correlation with the clinical features was estimated individually and then a cumulative correlation of all factors, i.e. clinical features, platelet counts and the cytokines was estimated. All data were analyzed using the statistical package for social science (SPSS) 10.0 for Windows program on the computer.

\section{Results and Discussion}

A total of 100 blood samples for this study were collected from 34 (42.5\%) pediatric patients and $46(57.5 \%)$ adult patients. Out of these 50 were male and 50 females. The patients were tested for NS1 antigen of DENV and IgM antibodies against DENV. Positive cases were then selected for the study as per their platelet count. All the patients included in the study had a platelet count of less than 1 , 00, 000 / cu.mm minimum count being 16000 and a maximum of $1,00,000$ with a mean value of 63,000 .

Clinical features of the study population were scored (based on the WHO classification of dengue with and without warning signs an arbitrary scoring was done) as depicted in Table 1.

It was found that the maximum number of pediatric patients did not present with significant clinical complications whereas the adult population showed bleeding manifestations in about one third of cases. The platelet counts of patients were compared to their clinical features by ANOVA test. There was a significant correlation found between the values and the clinical feature scores. The $F$ value computed was 2.909 with a significance of.040 $(<0.05)$ and thus there was a positive correlation between the platelet counts and the clinical features.
The IL-10 values were graded (Based on the study of Gathsaurie Neelika Malavige et al., $(2012,2013)$ an arbitory grading was done) as given in Table 2. The frequency of IL-10 values in patient population is depicted in Table 3. One third of the dengue positive patients had very high levels of IL-10 level. Interleukin 10 values and clinical features were analysed using Pearson's Chi Square Test and the Significance was calculated. There was a significant association $(\mathrm{p}=0.025)$ between the IL categorization and the clinical features i.e. patients with Severe Dengue or Dengue with danger signs had significantly higher number of IL-10 levels.

Immunity to Dengue virus (DENV)is serotype-specific and long term. However, secondary infection by a heterologous serotype is a pre-disposing factor for severe disease. This was attributed to the phenomenon of 'original antigenic sin'.

Primary dengue infection occurs when a person is infected with dengue virus for the first time with any one serotype. Months to years later, a more severe form of dengue illness may appear (called secondary dengue infection) due to infection with another serotype which is different from the first serotype causing primary infection (Wahala and Aravinda M. de Silva, 2011).

Antibody response against Dengue virus infection with dengue virus induces the production of both neutralizing and non neutralizing antibodies. The neutralizing antibodies are protective in nature. Such antibodies are produced against the infective serotype (which last lifelong) as well as against other serotype (which last for some time). Hence, protection to infective serotype stays lifelong but cross protection to other serotypes diminishes over few months. The non neutralizing antibodies last lifelong and are heterotypic in nature i.e. they are produced 
against other serotypes but not against the infective serotype. Such antibodies produced following the first serotype infection can bind to a second serotype, but instead of neutralizing the second serotype, it protects it from host immune system by inhibiting the bystander B cell activation antigen against the second serotype. The above phenomenon is called Antibody Dependent Enhancement which explains the reason behind the severity of secondary dengue infection. Being suboptimal in specificity and function, they fail to control infection and, instead, contribute greatly to a 'cytokine storm' (Wahala and Aravinda M. de Silva, 2011).

There were 34 pediatric and 45 adult patients included in the study. The male and female patients included in the study were equal. As stated by Deen et al and WHO definitions for Dengue 2009 severe dengue has been classified as dengue with warning signs and dengue without warning signs (Deen et al., 2006)This criteria was used to score the patients under four categories respectively.

Patients with a score of 1 and 2 showed signs and symptoms of dengue fever and were considered as Dengue without warning signs, however the ones with a score of 3 and 4 were the ones with danger signs and likely cases of severe dengue.

The frequency distribution of these features showed that most patients who tested positive for Dengue remained asymptomatic or had vague manifestations. This was found in around 37 percent of the cases. The common presenting symptom in patients with severe dengue was bleeding manifestations; which was found in around one third of the total cases. Patients with moderate dengue most commonly presented with typical features of pedal oedema, abdominal bloating etc. which could be likely due to low platelet counts accounting to the beginning of capillary leakage and associated pathology. A few cases also progressed to shock or DSS (Dengue Shock Syndrome) and Encephalitis; these being the hallmarks of severe Dengue.

Similar results were found in a study done by Priyadarshini et al., (2010) in North India where based on clinical features, $71.9 \%$ $(\mathrm{n}=159)$ of the patients were classified as DF, $50 \%$ of them had thrombocytopenia without any bleeding. Presence of any two of the DHF defining criteria by WHO, categorized 62 patients as DHF cases. Seven patients were defined as severe DHF (grades III/IV) with signs of circulatory failure. One fatal patient had severe thrombocytopenia, plasma leakage and circulatory failure (Grade IV). Fever with chills, headache, myalgia and nausea/vomiting were reported equally by DF and DHF patients. Joint pain, retroorbital pain and itching were observed in a significantly larger number of DF cases (p, 0.05, x2 test). Abdominal pain, maculopapular rash/petechiae and conjunctival congestion were found to be more prevalent in DHF cases (p, 0.05, x2 test) [The correlation between age and clinical features was calculation using Pearsons's Chi Square test. There was insignificant correlation between the two parameters; hence proving that age does not play any prognostic role in pathogenesis of severe dengue. This was also in correspondence to a similar study done by Priyadarshini et al., (2010).

The frequency of clinical features did not differ much in the two gender groups and the results were comparable. Similarly, when correlation using Chi Square was calculated ( $p$ $<0.025$ ) it was found to be insignificant showing there is possibly no significant correlation between gender and dengue prognosis. Similar results were depicted by Pandey et al., (2015) in their respective studies. 
Time and again it has been stated in various studies that the most severe complications following dengue fever are attributed due to plasma leakage occurring as a result of thrombocytopenia in patients with dengue. This is more severe in secondary dengue where non neutralizing antibodies are produced that lead to Antibody dependent Enhancement leading to antibodies against platelets leading to thrombocytopenia (Pandey et al., 2015).

The patients included in the study had platelet count ranging from 16000 to 100000 with a mean of 63000 . There was a significant correlation between the platelet count and the clinical features i.e. 0.04 ( $\mathrm{p}<0.05)$. This has also been stated by Rathakrishnan et al., (2012) in is study in a similar group of patients where the platelet counts were significantly decreased in patients with warning signs and moreover there was also a fall in the platelet count during the post febrile phase indicating a role of immune related mechanisms playing a pathophysiological role during the defervescence stage of Dengue (Rathakrishnan et al., 2012).

The IL10 values were graded based on their OD values (optical density) and these were compared with the respective standards to compute the value in $\mathrm{pg} / \mathrm{ml}$. Most dengue positive patients had IL 10 values above $50 \mathrm{pg} / \mathrm{ml} /$. In comparison to Pandey et al., 's study between cases(serologically tested positive for dengue $\operatorname{IgM}$ ) and controls (tested negative); the levels of serum IL10 values in control population was found below $10 \mathrm{pg} / \mathrm{ml}$. The number of such population in the study was one-fifth of all cases (Pandey et al., 2015).

The IL 10 levels were compared to clinical features and the correlation was computed by Pearson's Chi Square test. The value of IL 10 in patients with severe dengue that is in the patients with a score of 3 and 4 was significantly high. More than $70 \%$ of cases with clinical features of Dengue with warning signs had raised IL10 levels. However, it was found that there were around one third of cases where IL10 values were high when the patient's clinical score was 1 and 2 i.e. dengue without warning signs. These results were similar to those found by Pandey et al in their study where they have stated that levels of IL10 were significantly increased in the presence of Melina (a sign of gastrointestinal bleeding). High serum levels of antiinflammatory cytokines such as IL-10 were also found in DHF. Elevated levels of IL-10 in DHF patients compared to DF patients, reflecting an enhanced regulatory response to the more intense immune activation in DHF. The correlation between IL 10 and clinical features was calculated using Pearson's Chi square test which showed a significant relation between the two.

IL 10 estimation in patients of Dengue with warning signs showed a sensitivity of $100 \%$ and a very high positive predictive value. In addition to the extrinsic ADE pathway, in which the Fcy receptor directly facilitates DENV binding onto the cell surface for DENV infection/replication, an intrinsic ADE pathway induces IL-10-mediated immunosuppression. For the intrinsic pathway, the ADE of DENV infection triggers IL-10 production through an immune complex associated with the Fc $\gamma$ receptor to enhance the infection severity.

In the presence of $\mathrm{ADE}$, the $\mathrm{Fc} \gamma$ receptor can facilitate viral entry and trigger intracellular signaling. The generation of autoimmunity and ADE may cause concerns for vaccine development against DENV infection. Both viral particles acting through the extrinsic pathway and $F c \gamma$ receptor signaling through the intrinsic pathway are important for IL-10 induction. 
Studies done by Tsun Ting Tsai et al., show that overall, higher levels of IL-10 were detected in DHF/DSS patients compared with DF patients, and this trend was observed for infants, children, and adults. The time-kinetic analysis showed increased levels of IL-10 from the onset of fever to defervescence, and viremia primarily occurs during fever in dengue patients. The relationship between IL10 and viral replication is therefore speculated, and the possible pathogen effects may result from the IL-10-mediated inhibition of the antiviral IFN response. Maximal plasma IL-10 levels measured from the acute phase of infection correlated with the degree of plasma leakage, as determined by the pleural effusion index. Thus, IL-10 may cause lymphocyte dysfunction through the suppression of the $\mathrm{T}$ cell proliferative response to mitogens, which occurs in dengue patients during the early stages of infection. Furthermore, having thrombocytopenia, has been associated with the presence of IL-10. There has also been association found between elevated liver enzymes which could contribute to severe symptoms in Dengue to raise IL 10 levels (Tsun Ting Tsai et al., 2013).

Table.1 Frequency of clinical feature score

\begin{tabular}{|l|l|l|r|}
\hline S.NO & SIGNS AND SYMPTOMS & SCORE & Percent \\
\hline $\mathbf{1}$ & Clinical features- no bleeding, vague symptoms & 1 & 37.5 \\
\hline $\mathbf{2}$ & Abdominal pain, oedema, signs of dehydration & 2 & 22.5 \\
\hline $\mathbf{3}$ & Bleeding manifestations with danger signs & 3 & 28.8 \\
\hline $\mathbf{4}$ & Compensated shock & 4 & 11.3 \\
\hline
\end{tabular}

Table.2 Grading of IL-10 values

\begin{tabular}{|c|l|l|}
\hline S.NO & \multicolumn{1}{|c|}{ VALUES } & \multicolumn{1}{|c|}{ INTERPRETATION } \\
\hline $\mathbf{1}$ & 0 & Negative \\
\hline $\mathbf{2}$ & $0-10$ & Borderline positive \\
\hline $\mathbf{3}$ & $11-50$ & Moderately High \\
\hline $\mathbf{4}$ & $51-100$ & High \\
\hline $\mathbf{5}$ & $>100$ & Very high \\
\hline
\end{tabular}

Table.3 Frequency of IL-10 values in patient population

\begin{tabular}{|c|c|c|}
\hline $\begin{array}{l}\text { S.NO } \\
\cdot\end{array}$ & VALUES & $\begin{array}{c}\text { CUMULATIVE } \\
\text { PERCENT }\end{array}$ \\
\hline 1 & 0 & 1.3 \\
\hline 2 & $0-10$ & 16.3 \\
\hline 3 & $11-50$ & 51.3 \\
\hline 4 & $51-100$ & 70.0 \\
\hline 5 & $>100$ & 100.0 \\
\hline
\end{tabular}

Moreover, studies done in the past (Tsun Ting Tsai et al., 2013; Gathsaurie Neelika Malavige et al., 2012) show that the level of
IL-10 is higher in secondary DENV-infected patients than in primary DENV-infected patients. IL-10 induction is associated with 
severe DENV infection and is a potential biomarker for acute DENV infection. Specifically, IL-10 expression acts as predictive marker of death for DHF patient [With such a high sensitivity IL10 assay can prove to be an important screening marker for prognosis of Dengue Fever patients progressing to Severe Dengue (Gathsaurie Neelika Malavige et al., 2013).

In conclusion, early diagnosis and interventional measures can play a vital role in Dengue prognosis and can also prevent the patient from landing into Dengue Hemorrhagic Fever or Dengue Shock Syndrome. Moreover an insight into the Prognostic markers of Dengue can also help in early detection of Dengue with Warning Signs. The present study clearly indicates that IL10 is a highly sensitive marker of Severe Dengue and can be used as a screening tool in Secondary Dengue patients or those with warning signs.

\section{Acknowledgement}

The authors are gratefully acknowledge The Dean, Tirunelveli Medical College Hospital, Tirunelveli, Tamil Nadu and The Staff of Microbiology, Medicine and Paediatrics Department of Tirunelveli Medical College Hospital.

\section{References}

Cecilia, D. (2014). Current status of dengue and chikungunya in India. WHO SouthEast Asia J Public Health 3(1):22-27.

Deen, J.L., Harris, E., Wills, B., Balmaseda, A., Hammond, S.N., et al., (2006). The WHO dengue classification and case definitions: time for a reassessment. Lancet 8; 368(9530): 170-3.

Gathsaurie, N.M., Li-Chieh Huang, Maryam, S., et al., (2012). Cellular and Cytokine Correlates of Severe Dengue Infection.
PLoS ONE; 7(11).

Gathsaurie, N.M., Laksiri, G., Lukmall, A., Thashi, C., Maryam, S., Sachie, F., et al., (2013). Serum IL-10 as a marker of severe dengue infection. BMC Infectious Diseases.13:341

Halstead, S.B (2009). Controversies in dengue pathogenesis. Paediatr Int ChildHealth. 32(1): 5-9.

Martina, B.E, Koraka, P., Osterhaus, A.D. (2009). Dengue virus pathogenesis: an integrated view. Clin Microbiol Rev 22(4): 564-581.

Martin, J., Jenkins, R.H., Bennagi, R., Krupa, A., Phillips, A.O., Bowen, T. et al (2011). Post-transcriptional regulation of transforming growth factor beta-1 by microRNA-744. PLoS One, 6(10).

Monath, T. P. (1994). Dengue: the risk to developed and developing countries. Proc Natl Acad Sci USA 91, 23952400.

Natalia Houghton-Trivin, Doris. M., Salgado, Jairo, A. Rodr'1guez, Irene Bosch, Jaime, E., Castellanos et al., (2010). Levels of soluble ST2 in serum associated with severity of dengue due to tumour necrosis factor alpha stimulation. Journal of General Virology 91:697-706.

Nidhi Pandey, Amita. J., Garg, R.K., Rashmi, K., Agrawal, O.P., Lakshmana Rao, R.K. (2015). Serum levels of IL-8, IFNc, IL-10, and TGF $b$ and their gene expression levels in severe and nonsevere cases of dengue virus infection. Arch Virol 160:1463-147

Priyadarshini, D., Gadia, R.R., Tripathy, A., Gurukumar, K.R., Bhagat, A., et al., (2010). Clinical findings and proinflammatory cytokines in dengue patients in Western India: a facilitybased study. PLoS One5: 8709.

Rathakrishnan, A., Wang, S.M., Hu, Y., Khan, A.M., Ponnampalavanar, S., et al., (2012). Cytokine Expression Profile 
of Dengue Patients at Different Phases of Illness. PLoS ONE 7(12): e52215. doi:10.1371/journal.pone.0052215

Tillu, H., Tripathy, A.S., Reshmi, P.V., Cecilia, D (2015). Altered profile of regulatory $\mathrm{T}$ cells and associated cytokines in mild and moderate Dengue Eur J Clin Microbiol Infect Dis. 35:453-461.

Tsung, T.T., Yi-Jui Chaung., Yee- Shin Lin. et al., (2013). An emerging role for the anti- inflammatory cytokine interleukine-10 in dengue virus infection. Journal of biomedical Science. 20:40

Wahala, M. P. B., Wahala and Aravinda M. de Silva (2011). The Human Antibody Response to Dengue Virus Infection. doi: 10.3390/v3122374.3(12): 23742395.

World Health Organization (WHO) (2009). Dengue and dengue haemorrhagic fever. Available online at: http://www.who.int.

\section{How to cite this article:}

Saishruti, G. Sucila Thangam and Revathy. 2019. Pathophysiologic and Prognostic Role of IL10 as a Proinflammatory and Regulatory Cytokine in Dengue Fever. Int.J.Curr.Microbiol.App.Sci. 8(01): 1779-1787. doi: https://doi.org/10.20546/ijcmas.2019.801.189 